\title{
Application of Music Therapy in Intervention Research of Autistic Children
}

\author{
Ting Wang ${ }^{1, a}$, Taifang Chen ${ }^{2, b}$, Jinwen Wang*3,c \\ ${ }^{1}$ School of Humanities, Jiangxi University of Traditional Chinese Medicine, Nanchang, China \\ ${ }^{2}$ School of Humanities, Jiangxi University of Traditional Chinese Medicine, Nanchang, China \\ ${ }^{3}$ School of Public Administration, Nanchang University, Nanchang, China
}

\begin{abstract}
With the continuous development of China's information technology, medical information is also constantly innovating, which is conducive to the diversity and effectiveness of the treatment of various diseases, including autism. Music therapy is the embodiment of the innovation of information media in medical informationization. Music therapy is a kind of information communication medium that uses music to stimulate and hypnotize people and stimulate physical reactions in various forms of music activities to promote people's physical and mental health. It is often used in the intervention research of autism. This article summarizes the effectiveness of music therapy for children with ASD in the latest research at home and abroad, and the advantages of music therapy in promoting social communication, improving attention, reducing stereotyped behavior, and improving negative emotions in children with ASD. It also reveals music How the treatment can improve the facial emotion recognition ability of children with ASD, the role of assistive robots and family participation in the music therapy of children with ASD.
\end{abstract}

\section{Introduction}

Music carries and conveys rich meaning just like language. Originated in the 20 mid-century music therapy (Music Therapy) is music in modern life in a new application form ${ }^{[1]}$. Music therapy is different from ordinary drug therapy. It can promote physical and mental health, relieve stress, relieve pain, and help express emotions, enhance memory, and improve communication. It has become a new hot spot in current domestic and foreign research ${ }^{[2]}$.

With autism spectrum disorders (Autism Spectrum disorder, ASD) the increasing incidence ${ }^{[3]}$, music therapy in improving ASD adverse symptoms played a role attracted much attention. Wan Xuan and other researchers through analysis of past literature, found that ASD is not only exhibited a strong musical pitch perception ability, but also reflected in the music aspect of a certain advantage ${ }^{[4]}$. Therefore, music therapy is recommended as an effective intervention method for improving and enhancing the social interaction, language communication and emotional expression ability of ASD children ${ }^{[5]}$. This study will ASD children actually need starting to explore music therapy in ASD children applied research interventions, as well as music therapy for ASD children effectiveness of interventions, and to further explore the latest researches in music therapy for ASD children's club cross communication, attention, stereotyped behavior, mood and emotion to enhance the role of other aspects.

\section{Intervention goals of music therapy}

\subsection{Social interaction and communication}

ASD social interaction and communication disorders in children notable performance

in language terms, however, recent studies have shown that ASD musical behavior of children involved in the close link between skills and language

development ${ }^{[6]}$. Music as a medium greatly increased the ASD proactive social participation of children and promote their language and other social skills ${ }^{[7-8]}$.

There is a connection between music training and language comprehension ${ }^{[9]}$. The research on music therapy to improve the language disorder of children with ASD can be roughly divided into two categories. One is from passive to active music therapy, and the other is the alternate intervention of music therapy and non-music therapy ${ }^{[10-11]}$. The two types of music therapy methods have a good intervention effect on the social interaction and communication skills of children with ASD. Foreign recent empirical studies exposing shows a music therapy to promote ASD effectiveness of children's language development, this discovery is given for music therapy on physiological aspects of ASD children social interaction and communication evidence obstacles to improved $^{[12]}$. (Table1)

\footnotetext{
a125323611@qq.com,b524756132@qq.com

c* Corresponding author: 1486523178@qq.com
} 


\begin{tabular}{|c|c|c|c|c|c|c|c|c|c|}
\hline \multirow[t]{3}{*}{ Outcomes } & \multicolumn{6}{|c|}{ Observed values } & \multicolumn{3}{|l|}{ Effect size } \\
\hline & \multicolumn{3}{|c|}{ Music } & \multicolumn{3}{|c|}{ Non-music } & \multirow[t]{2}{*}{ Mean difference } & \multirow[t]{2}{*}{ $\pm 95 \%$ Cl } & \multirow[t]{2}{*}{ Standardized effect size ( $d$ ) } \\
\hline & $n$ & Mean & $\pm 95 \% \mathrm{Cl}$ & $n$ & Mean & $\pm 95 \% \mathrm{Cl}$ & & & \\
\hline \multicolumn{10}{|l|}{ Primary autcomes } \\
\hline $\mathrm{CCC}-2$ & & & & & & & 4.84 & 4.08 & 0.34 \\
\hline Baseline & 25 & 76.84 & 5.64 & 23 & 77.65 & 5.45 & & & \\
\hline Post-intervention & 24 & 80.46 & 6.43 & 23 & 76.43 & 5.02 & & & \\
\hline Changes from baseline & 25 & 3.62 & 0.78 & 23 & -1.22 & -0.43 & & & \\
\hline SRS-11 & & & & & & & 0.65 & 3.45 & 0.06 \\
\hline Baseline & 26 & 70.15 & 3.68 & 25 & 72.24 & 4.47 & & & \\
\hline Post-intervention & 26 & 69.36 & 4.39 & 25 & 70.8 & 3.98 & & & \\
\hline Changes from baseline & 26 & -0.79 & 0.71 & 25 & -1.44 & -0.49 & & & \\
\hline PPVT-4 & & & & & & & 0.03 & 4.35 & 0.00 \\
\hline Baseline & 26 & 94.57 & 10.05 & 25 & 85.48 & 11.52 & & & \\
\hline Post-intervention & 26 & 95.04 & 10.66 & 25 & 85.92 & 12.11 & & & \\
\hline Changes from baseline & 26 & 0.47 & 0.61 & 25 & 0.44 & 0.59 & & & \\
\hline \multicolumn{10}{|l|}{ secondary outcomes } \\
\hline FQOL & & & & & & & 7.06 & 6.27 & 0.57 \\
\hline Baseline & 26 & 102.42 & 5.25 & 25 & 104.08 & 539 & & & \\
\hline Post-intervention & 26 & 10536 & 3.86 & 25 & 99.96 & 4.65 & & & \\
\hline Changes from baseline & 26 & 2.94 & -1.39 & 25 & -4.12 & -0.74 & & & \\
\hline VABS-MB & & & & & & & 0.08 & 0.65 & 0.04 \\
\hline Baseline & 26 & 19.8 & 0.59 & 24 & 20 & 0.74 & & & \\
\hline Post-intervention & 26 & 19.42 & 0.71 & 24 & 19.54 & 0.86 & & & \\
\hline Changes from baseline & 26 & -0.38 & 0.12 & 24 & -0.46 & 0.12 & & & \\
\hline
\end{tabular}

\subsection{Attention}

Attention deficit is considered to be one of the earliest manifestations of social deficits in children with ASD. The current attention studies of children with ASD at home and abroad cover selective attention, sustained attention, and joint attention [13]. Music therapy is usually used to improve children with ASD. Common attention. Music can effectively attract the attention of children with ASD, especially joint attention. Thibeault found that a 12-month impromptu music therapy can effectively improve the common attention deficit of children with ASD from 1 to 6 years old ${ }^{[14]}$. Compared with other children, music therapy Treatment can make ASD children more focused.

Visual music therapy is a new method to improve the attention of children with ASD. Visual music therapy based on audiovisual sensory integration technology integrates music, pictures, lighting and other elements. Using multi-sensory stimulation to attract the attention of children with ASD is a new development in music therapy. Researchers found that the intervention of attention problems in children with ASD through visual music therapy stimulated the corresponding emotional experience of children with ASD and improved their receptivity. Visual music therapy using songs and familiar stories can enhance ASD children's attention, help ASD children pay more attention to their faces, and promote ASD children to participate in social interaction more naturally and spontaneously. It is to help ASD children in a natural situation An effective way to interact with others $[15]$.

\subsection{Stereotyped behavior}

Stereotyped behavior is a clinical feature that cannot be ignored in children with ASD, including behaviors such as stereotyped repetitive motion, object use, and language use.

Research by Khanzadeh et al. has shown that music therapy can promote ASD children to concentrate on participating in interaction, thereby increasing appropriate social behaviors and reducing inappropriate repetitive stereotyped behaviors [16]. Rhythm-based, object-free music therapy allows ASD children to actively participate in creative experience activities (including listening, singing, playing, creating, etc.), so as to achieve therapeutic goals ${ }^{[17]}$.

\subsection{Emotions}

Emotional disorders in children with ASD are mainly manifested as poor emotional expression and impaired regulation of emotional and emotional states ${ }^{[18]}$. Due to impaired language function, children with ASD need to express their thoughts through emotions and emotions. Therefore, a large number of researchers have focused on the emotions of ASD children. Difficulty in expressing emotions intervened.

The research results of Brian, Stephen, James and others show that music therapy has a positive effect on the emotional and emotional intervention of ASD children, which can relieve the anxiety and depression of ASD children and make them show more positive emotions ${ }^{[19]}$.One intervention is to directly change the emotional emotions of children with ASD to reduce negative emotions [20-21]; the other intervention is to improve the social skills of children with ASD through music experience activities so that they feel a certain degree of confidence and sense of accomplishment, Thereby indirectly changing negative emotions ${ }^{[22]}$. The results found that music therapy can have a positive effect on children with ASD. After ASD children receive music therapy, negative emotions show a downward trend in the 
early and late stages of treatment, and positive emotions show an upward trend.

\section{The role of music therapy}

With the in-depth study of music therapy for children with ASD, and the continuous integration of emerging science and technology with music therapy in recent years, "Music therapy enhances the ability of ASD children's face and emotion recognition", "The role of assistive robots and family participation in music therapy for ASD children "'" and other research fields have attracted more and more attention from domestic and foreign researchers and have achieved more research results.

\subsection{Music therapy improves facial emotion recognition ability of ASD children}

Facial emotion recognition (FER) obstacles in children with ASD refer to specific facial processing defects (such as seeing only part of the face instead of the whole) or difficulty in facial emotion recognition (such as longer reaction time or inability to recognize) ${ }^{[23]}$.

Many children with ASD have difficulty perceiving facial emotions, but can recognize emotions in music. Music therapy can improve their facial emotion recognition ability. The researchers tested the ability of ASD children to recognize facial emotions under different music types, and the results showed that happy background music can improve the emotion recognition of happy faces, and sad background music can improve the emotion recognition of sad faces ${ }^{[24-25]}$. After music therapy, some children with ASD can recognize facial emotions and use their body movements or facial muscles to imitate and express facial emotions. Background music can be an effective tool to increase facial emotion recognition for ASD children ${ }^{[26-27]}$. It can be seen that music is an effective channel for emotional communication with ASD children.

\subsection{The role of assistive robots in music therapy for children with ASD}

In recent years, the application of social assistance robots (SAR) in music therapy for children with ASD has gradually increased. The process of robot-assisted music therapy for children with ASD is simple and predictable, and it is easy to attract children with ASD to participate. Imitation plays a key role in the development of children's social skills. Studies have shown that music therapy with the assistance of robots greatly promotes the imitation ability of children with ASD, thereby increasing their social interaction behavior ${ }^{[28]}$.

Researchers use human-shaped social robots (NAO) to perform human-computer interaction music therapy for children with ASD, focusing on the joint attention skills of children with ASD, thus laying the research foundation for NAO to perform music intervention tasks autonomously [29]. The latest intervention research, based on musicassisted scenes, systematically teaches music to ASD children by a new type of robot (NAO). After NAO robot- assisted music therapy, the social and cognitive abilities of ASD children are generally improved ${ }^{[30]}$. It can be seen that assistive robots provide a new way of interaction for music therapy. As a tool and facilitator, assistive robots can improve the music performance experience of children with ASD, and indeed have the ability to promote the development of children with ASD in music teaching and cognitive learning ${ }^{[31]}$.

\subsection{The role of family participation in music therapy for children with ASD}

Commonly used music therapy usually targets individuals with ASD, ignoring the role of family. Intervention studies that shift the focus of music therapy to the family have shown that parents' active participation in music therapy courses can promote the development of ASD children and families in a positive direction ${ }^{[32]}{ }^{[33]}$.

Family-centered music therapy breaks through the traditional treatment methods, and the treatment results are more optimistic. In a family-centred music therapy (FCMT) intervention study, Thompson et al. supported and instructed parents to interact with their children in music creation activities. After intervention, the ASD children's social skills in the family were improved. Behaviors in social activities outside the family are also more stable ${ }^{[34]}$. In addition, studies have shown that FCMT is based on the conceptual framework of music intervention involving parental participation. It takes the family as the center and systematically trains parents to learn and use personalized music intervention methods in daily environments such as families and communities. Parents are responsible for some or all of the interventions Training to reduce problem behaviors in children with ASD or improve social communication skills ${ }^{[35-36]}$. FCMT is currently one of the most useful strategies to promote the growth of ASD children, meeting the emotional needs of ASD children and their parents, thereby enhancing family satisfaction.

\section{4 conclusion}

After reviewing the literature, this article confirms that music therapy has a positive impact on children with ASD, improves social communication, attention, emotional and emotional abilities of children with ASD, and improves stereotypes and other bad behaviors. This shows that music therapy for children with ASD is valuable Intervention research. Music is a unique and artistic signal stimulus. Music therapy has no side effects and is low cost. In this regard, this article provides a series of enlightenments for ASD children's educational practice based on music therapy. Such as: First, use music therapy as a routine education and rehabilitation training method for children with ASD. Second, the combination of assistive robot technology and music therapy is applied to the education of children with ASD. Third, encourage and guide parents to participate in rehabilitation training for ASD children.

However, there are still some shortcomings in this article: First, the current evaluation of music therapy in 
children with ASD mostly relies on scale evaluation or expert evaluation, with a single evaluation method; second, the neural mechanism of music therapy in ASD children has not been further clarified. Therefore, future research needs to conduct more diversified, objective quantitative evaluations and other behavioral experiments, physiology and imaging methods to explore and verify the neural basis and related mechanisms of music therapy.

\section{Acknowledgment}

This research was financially supported by Jiangxi Social Science Foundation Project of China(20JY20) and Jiangxi Provincial planning of educational sciencegeneral project of China(19YB015).

\section{References}

1. Zhou Linshu, Jiang Cunmei. The cognitive neural mechanism of music conveying philosophical concepts. Advances in Psychological Science, 2016, 24(6): 855-862

2. Reschke-Hernández A E. History of music therapy treatment interventions for children with autism. Journal of Music Therapy, 2011, 48(2): 169-207

3. Newschaffer C J, Croen L A, Daniels J, Giarelli E, Grether J K, \& Levy S E, et al. The epidemiology of autism spectrum disorders. Annu Rev Public Health, 2010, 8(3): 151-161

4. Wan Xuan, Dong Shihua, Jiang Cunmei. The processing of music, speech and phonology by autistic people. Psychological Science, 2014, 37(1): 217-224

5. Kim J, Wigram T, Gold C. The effects of improvisational music therapy on joint attention behaviors in autistic children: A randomized controlled study. Journal of autism and developmental disorders, 2008, 38(9): 1758-1766

6. Vaiouli P, Andreou G. Communication and language development of young children with autism: A review of research in music. Communication Disorders Quarterly, 2018, 39(2): 323-329

7. Gattino G S, Riesgo R S, Longo D, et al. Effects of relational music therapy on communication of children with autism: A randomized controlled study. Nordic Journal of Music Therapy, 2011, 20(2): 142154

8. Chang X, Liu Y, Wang P, et al. The effects of music interventions on verbal expression in children with autism. Advances in Psychological Science, 2016, 24(9): 1391-1397

9. Wang Hang, Jiang Jun, Jiang Cunmei. The influence of music training on cognitive ability. Advances in Psychological Science, 2015, 25(3): 419-429

10. Finnigan E, Starr E. Increasing social responsiveness in a child with autism: A comparison of music and non-music interventions. Autism, 2010, 14(4): 321348
11. Fong C E, Jelas Z M. Music education for children with autism in Malaysia. Procedia-Social and Behavioral Sciences, 2010, (9): 70-75

12. Sharda M, Tuerk C, Chowdhury R, et al. Music improves social communication and auditory-motor connectivity in children with autism. Translational Psychiatry, 2018, 8(1): 1-13

13. Bhat A N, Landa R J, Galloway J C. Current perspectives on motor functioning in infants, children, and adults with autism spectrum disorders. Physical Therapy, 2011, 91(7): 1116-1129

14. Thibeault C A. Addressing Joint Attention Deficits with Children with Autism Spectrum Disorder: A Music Therapy Intervention Research Study. Masters thesis. Concordia University, 2017

15. Thompson $\mathrm{G} A$, Abel L A. Fostering spontaneous visual attention in children on the autism spectrum: A proof-of-concept study comparing singing and speech. Autism Research, 2018, 11(5): 732-737

16. Khanzadeh H, Ali A, Imankhah F. The effect of music therapy along with play therapy on social behaviors and stereotyped behaviors of children with autism. Practice in Clinical Psychology, 2017, 5(4): 251-262

17. Bhat A N, Srinivasan S. A review of "music and movement" therapies for children with autism: Embodied interventions for multisystem development. Frontiers in Integrative Neuroscience, 2013, (7): 22

18. Maskey M, Warnell F, Parr J R, et al. Emotional and behavioural problems in children with autism spectrum disorder. Journal of Autism \& Developmental Disorders, 2012, 43(4): 851-859

19. Boyd B A, Mcdonough S G, Bodfish J W. EvidenceBased behavioral interventions for repetitive behaviors in autism. Journal of Autism and Developmental Disorders, 2012, 42(6): 1236-1248

20. Rapp J T, Vollmer T R. Stereotypy I: A review of behavioral assessment and treatment. Research in Developmental Disabilities, 2005, 26(6): 527-547

21. Loftin R L, Odom S L, Lantz J F. Social Interaction and Repetitive Motor Behaviors. Journal of Autism and Developmental Disorders, 2008, 38(6): 11241135

22. Diehl J J, Schmitt L M, Villano M, et al. The clinical use of robots for individuals with autism spectrum disorders: A critical review. Research in Autism Spectrum Disorders, 2012, 6(1): 249-262

23. Lack M H, Chen N M, Iyer K K, et al. Mechanisms of facial emotion recognition in autism spectrum disorders: Insights from eye tracking and electroencephalography. Neuroscience \& Biobehavioral Reviews, 2017, 80: 488-515

24. Bruyn L D, Moelants D, Leman M. An embodied approach to testing musical empathy in subjects with an autism spectrum disorder. Music and Medicine, 2011, 4 (1): 28-36

25. Golan O, Gordon I, Fichman K, et al. Specific patterns of emotion recognition from faces in children with ASD: Results of a cross-modal matching 
paradigm. Journal of autism and developmental disorders, 2018, 48(3): 844-852

26. Katagiri J. The effect of background music and song texts on the emotional understanding of children with autism. Journal of Music Therapy, 2009, 46(1): 15-31

27. Petric F, Miklić D, Cepanec M, et al. Functional imitation task in the context of robot-assisted autism spectrum disorder diagnostics: Preliminary investigations. Proceedings of the 26th IEEE International Symposium on Robot and Human Interactive Communication (RO-MAN 2017), 2017, 12: $1471-1478$

28. Kossyvaki L, Curran S. The role of technologymediated music-making in enhancing engagement and social communication in children with autism and intellectual disabilities. Journal of Intellectual Disabilities, 2020, 24(1): 118-138

29. Duquette A, Michaud F, Mercier H. Exploring the use of a mobile robot as an imitation agent with children with low-functioning autism. Autonomous Robots, 2008, 24(2): 147-157

30. Zhang Xinxin, Wang Fang, Yang Guangxue. Research progress in the application of robotics in the education of children with autism spectrum disorders. China Special Education, 2018, 221(11):26-34

31. Thompson G A. Family-centered music therapy in the home environment: Promoting Johnston D, Egermann H, Kearney G, et al. Innovative Computer Technology in music based interventions for individuals with autism-Moving beyond traditional interactive music therapy techniques. Cogent Psychology, 2018, 5(1):1-18

32. Thompson G A, Mcferran K S, Gold C. Familycentred music therapy to promote social engagement in young children with severe autism spectrum disorder: A randomized controlled study. Child: Care, Health and Development, 2014, 40(6): 840-852

33. Hernandez-Ruiz E. Parent-Mediated Music Interventions with Children with ASD: a Systematic Review. Review Journal of Autism and Developmental Disorders, 2020, 5: 1-18

34. Pasiali V. The use of prescriptive therapeutic songs in a home-based environment to promote social skills acquisition by children with autism: Three case studies. Music Therapy Perspectives, 2014, 22 (1): 11-20

35. Hernandez-Ruiz, E. Parent coaching of music interventions for children with ASD: A conceptual framework. Nordic Journal of Music Therapy, 2019, (8): $1-22$

36. Xu Yun, Xu Chenxin, Zhu Minrui. The application of parent executive intervention in the families of children with autism. Chinese Journal of Clinical Psychology, 2019, 27(3): 634-637 